\title{
Une influence positive sur la qualité des soins
}

La médecine passe du corps individuel au collectif et revient de celui-ci à celui-là, par les chemins de la recherche et de l'Administration. Résultat: s'accroît régulièrement l'espérance de vie, jusqu'à ce chiffre récent, proprement incroyable, d'un trimestre par an. La population vieillit: ce verbe n'a plus le même sens. Non seulement change le corps des individus, mais l'allure de la Société. Michel Serres, Hominescence, Editions Le Pommier 2001, p. 20.

La soumission au processus de consultation des directives médico-éthiques intitulées «Traitement et prise en charge des personnes âgées en situation de dépendance» publiée pour la première fois à mi-juin 2003 dans le Bulletin des Médecins Suisses a suscité un grand intérêt. Elle avait été précédée d'une conférence de presse qui a éveillé un grand écho dans les médias. Une cinquantaine de prises de position individuelles ou provenant d'institutions de divers domaines et disciplines sont parvenues à l'Académie et ont été examinées avec le plus grand soin par les membres de la sous-commission en charge de la rédaction du document. Dans l'ensemble, les directives ont été considérées comme bienvenues et d'actualité, allant dans la bonne direction en soulignant le droit des personnes âgées dépendantes au respect de leur dignité, de leur autonomie et de leurs autres droits fondamentaux attachés à leur personne. Beaucoup de personnes attendent de ces directives qu'elles aient une influence positive sur la qualité des soins apportés aux personnes âgées. Certains pensaient que les directives allaient trop loin dans le détails des recommandations adressées aux Institutions, d'autres au contraire qu'elles n'allaient pas assez loin. Dans sa présentation le Prof. Andreas Stuck, président de la sous-commission en charge de rédiger ces directives, résume et répond aux remarques reçues.

Le rôle des directives et recommandations de l'Académie est de fixer des conditions minimales à respecter et de fixer des priorités et des buts, laissant aux responsables le soin de veiller à la stratégie à suivre pour les atteindre par la mise en place de réformes, si nécessaires, en fonction des moyens à disposition et d'établir un plan de développement des structures et des soins à l'intention des Autorités de surveillance. L'Académie ne saurait s'immiscer dans la réalisation de ces réformes, déjà du fait de la grande disparité d'une Institution à l'autre. Dès lors elle veille à bien séparer les directives à l'adresse du corps médical, des équipes des soins infirmiers et des divers thérapeutes, des recommandations à l'adresse des Institutions.

Le problème délicat des mesures de contrainte, limitatives de liberté, parfois nécessaires, seront traitées en détails dans de nouvelles directives de l'Académie en cours d'élaboration, mais déjà ici il est le sujet d'un chapitre particulier. De même, un chapitre est dévolu à l'attitude attendue en cas d'observation de maltraitance ou de négligence dans les soins. Les recommandations à l'intention des Institutions reprennent un par un tous les points des directives, mais cette fois pour inciter les Institutions à établir des normes internes et se fixer des objectifs dans l'adaptation et l'amélioration des conditions cadre et à veiller à la formation continue du personnel soignant.

Nous aimerions remercier à cette occasion, au nom de l'ASSM, les membres de la sous-commission, et plus particulièrement son Président, pour leur travail considérable. Nous espérons que ces nouvelles directives et recommandations trouveront la considération qu'elles méritent. A cet égard, l'Association Suisse des Infirmières et Infirmiers souhaite que ces directives et recommandations soient observées et appliquées, ce qui est en soi un indice encourageant.

Pour terminer, nous invitons à méditer cet autre extrait de l'ouvrage de Michel Serres: Comment se fait-il que le vieillissement de la population paraisse à des yeux exclusivement formés à l'économie comme l'affaiblissement d'un groupe, alors qu'il y favorise l'éducation, la culture et l'advenue d'une sagesse que la perspective seulement économique oublie au point de ne retenir de la vie humaine que ce qui ne vaut pas d'être vécu? (op. cité, page 227)

Prof. Michel B. Vallotton, Président de la Commission Centrale d'Ethique de l'ASSM

Prof. Werner Stauffacher, Président de l'ASSM 